\title{
New Trends on the Boron-Doped Diamond Electrode: From Fundamental Studies to Applications
}

\author{
Giancarlo Richard Salazar Banda, ${ }^{1}$ Yasuaki Einaga, ${ }^{2}$ \\ and Carlos Alberto Martinez-Huitle ${ }^{3}$ \\ ${ }^{1}$ Instituto de Tecnologia e Pesquisa/Programa de Pós-Graduação em Engenharia de Processos, Universidade Tiradentes, 49032-490,
Aracaju, SE, Brazil
${ }^{2}$ Department of Chemistry, Faculty of Science and Technology, Keio University, 3-14-1 Hiyoshi, Yokohama 223-8522, Japan
${ }^{3}$ Universidade Federal do Rio Grande do Norte, Centro de Ciências Exatas e da Terra. Departamento de Química, 59078-970 Natal,
RN, Brazil
}

Correspondence should be addressed to Giancarlo Richard Salazar Banda, gianrsb@gmail.com

Received 29 September 2011; Accepted 29 September 2011

Copyright (C) 2012 Giancarlo Richard Salazar Banda et al. This is an open access article distributed under the Creative Commons Attribution License, which permits unrestricted use, distribution, and reproduction in any medium, provided the original work is properly cited.

Boron-doped diamond (BDD) is presently the subject of considerable interest as an electrode material. This is a consequence of its very wide potential window in aqueous solution (ca. $\sim 3 \mathrm{~V}$ ), low background currents, resistance to thermal shock, extreme electrochemical stability, and corrosion stability in aggressive media. Diamond electrodes have indeed the largest potential window so far measured in aqueous electrolytes. This makes them also totally different to common electrode materials such as gold, platinum, or mixed metal oxide DSA-type electrodes.

Because of their unique electrochemical properties, doped diamond electrodes can be used for a plethora of applications. Since their properties largely differ from those of conventional electrode materials, in most cases, they will not replace these materials but will make electrochemical processes that were impossible before possible. Because of their wide range of applications, studies to contribute to the advance of the fundamental knowledge of this material are strongly important for their further technological application.

The electrochemistry of diamond has developed rapidly in recent years and is growing with the development of many practical applications of diamond electrodes, which impact almost every aspect of electrochemistry, from electroanalysis to industrial applications.

Several analytes can be oxidized or reduced by conducting diamond compared to other carbon-based materials before the breakdown of water in aqueous electrolytes. This is important for detecting and/or identifying species in solution since oxygen and hydrogen evolution do not interfere with the analysis. Thus, conductive diamond electrodes take electrochemical detection into new areas and extend their usefulness to analytes which are not feasible with conventional electrode materials.

This special issue initially contains four papers concerning the use of BDD electrode surfaces in the electrochemical detection of analytes using different techniques. Initially, bare BDD surfaces were used for the separation and determination of sulfonamides using a fully automated online solid phase extraction coupled with sequential injection analysis and high-performance liquid chromatography. The use of modified BDD surfaces with $\mathrm{Cu} / \mathrm{Pd}$ or $\mathrm{Au}$ for the detection of nitrate or $\mathrm{Se}^{4+}$, respectively, is also reported, as well as the use of glucose-oxidase-cobalt phthalocyanine-modified BDD electrode, without any additional electron mediator in the electrolyte solution, for the electrochemical detection of glucose.

The use of BDD surfaces for the electrochemical oxidation or combustion of pollutants from water is summarized and discussed in three papers. The first of these is a review article on the environmental application of BDD electrodes for electrochemical incineration of azo dyes in synthetic and real wastewaters. The other two papers deal with experimental results on the benzene oxidation in $0.5 \mathrm{M} \mathrm{K}_{2} \mathrm{SO}_{4}$ aqueous 
solution studied by cyclic voltammetry and electrochemical impedance spectroscopy and with the study of the BDD capacity to generate hydroxyl radicals that are not consumed in the adsorption layer and are available to oxidize other organic or inorganic species.

Four other review articles related to (i) the several important advances in the synthesis of boron-doped diamond thin films, especially those synthesized via gas-phase manipulation, (ii) the uses of BDD for energy conversion systems like fuel cells and rechargeable batteries, (iii) the current development status of nanocrystalline diamond electrodes for electrochemical applications, and (iv) recent advances in the use of BDD electrodes with different surfaces including nanostructured surface and chemically modified surface for the construction of various electrochemical biosensors are also reviewed in this special issue. Thus, several subjects from the synthesis to the application of BDD materials in electroanalysis, wastewater treatment, oxidants production, energy conversion systems, and nanodiamond electrochemistry are covered in the special issue.

In addition the synthesis of $\mathrm{N}$-doped hydrogenated amorphous carbon (DLC) thin films, oxygen plasma treated, with high electrochemical reactivity and stability is reported in one paper. The N-doped DLC thin films exhibited a wide working potential range over $3 \mathrm{~V}$, low double-layer capacitance, and reversible electron transfer kinetics for inorganic redox analytes, $\left[\mathrm{Fe}(\mathrm{CN})_{6}\right]^{3-/ 4-}, \mathrm{Ru}\left(\mathrm{NH}_{3}\right)_{6}^{2+/ 3+}$, and $\mathrm{Fe}^{2+/ 3+}$, which were on the same level as those of BDD.

Finally, we thank the authors for their contributions to this special issue and also commend the reviewers for their constructive and timely reviews. It is our hope that this issue will stimulate new ideas and expand further the growing area of BDD electrochemistry, for the benefit of electrochemists, engineers, and the broader scientific community.

Giancarlo Richard Salazar Banda
Yasuaki Einaga
Carlos Alberto Martinez-Huitle 


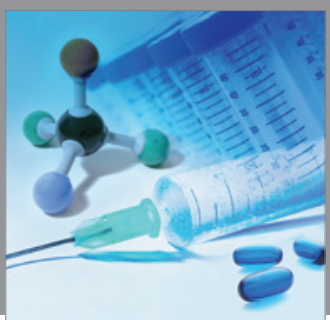

International Journal of

Medicinal Chemistry

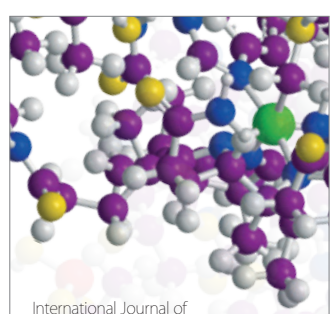

Carbohydrate Chemistry

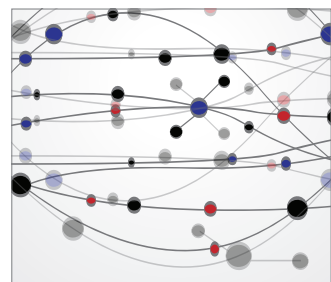

The Scientific World Journal
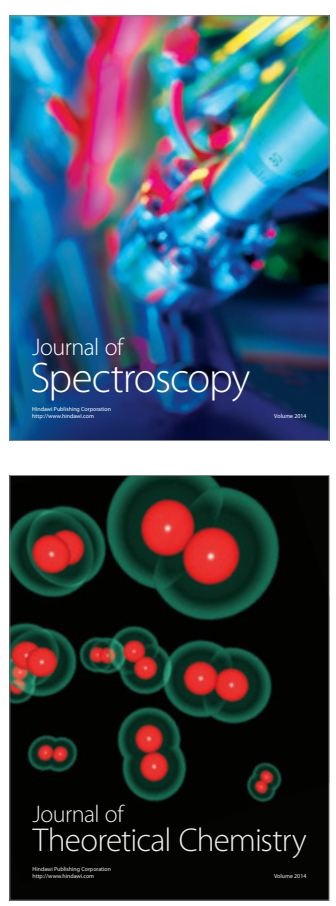
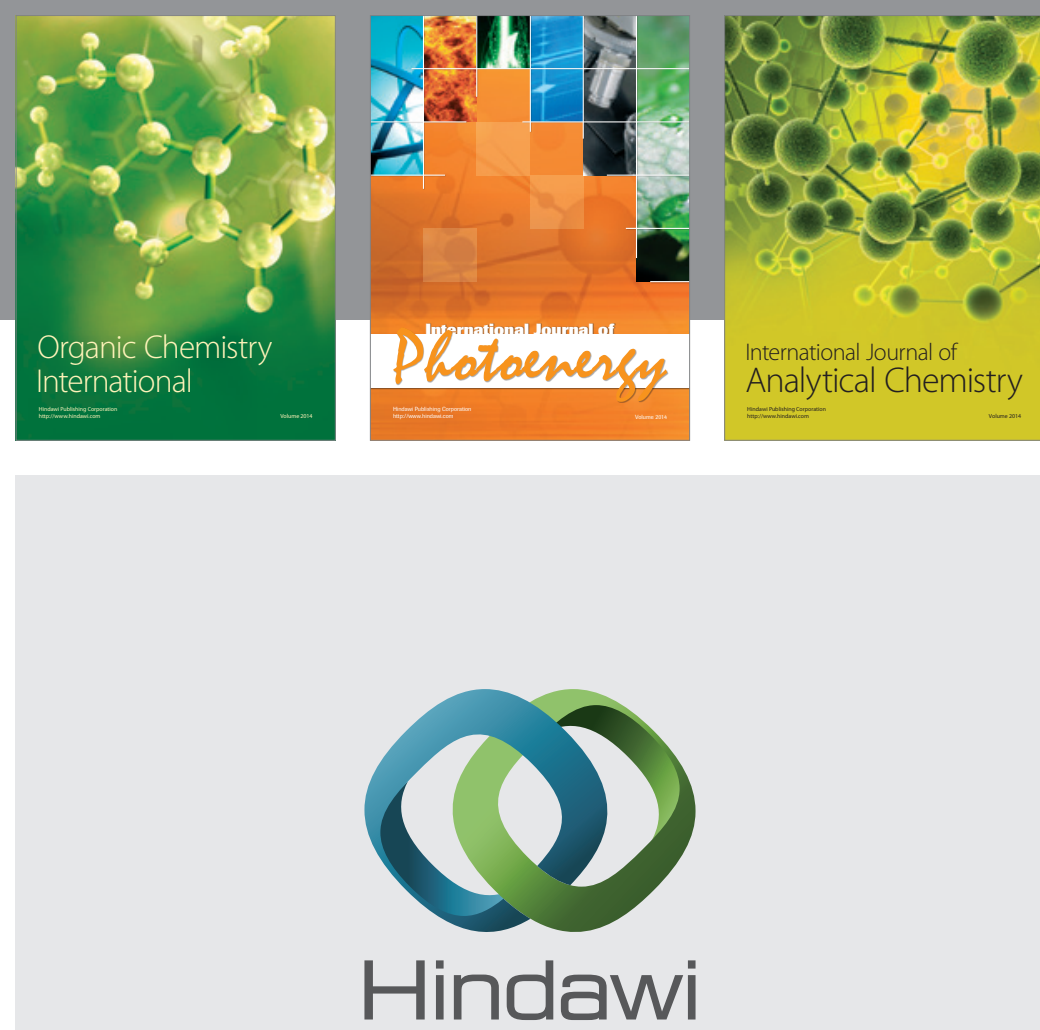

Submit your manuscripts at

http://www.hindawi.com
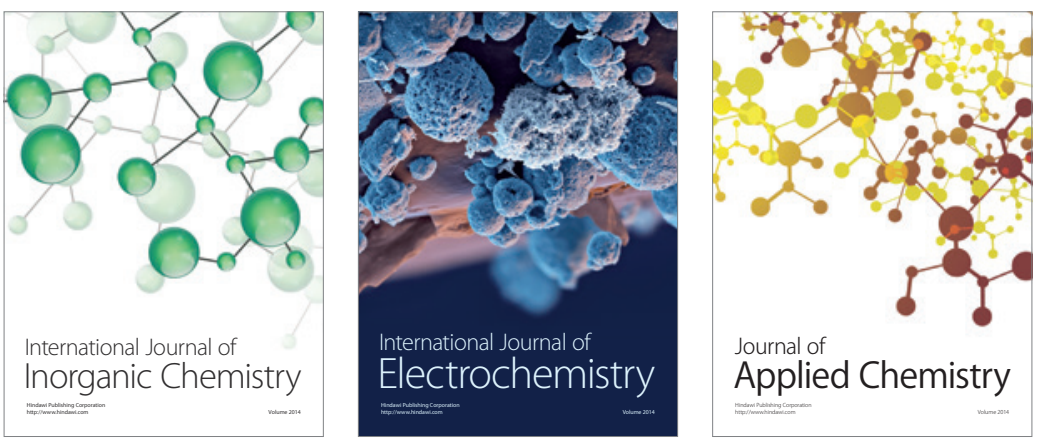

Journal of

Applied Chemistry
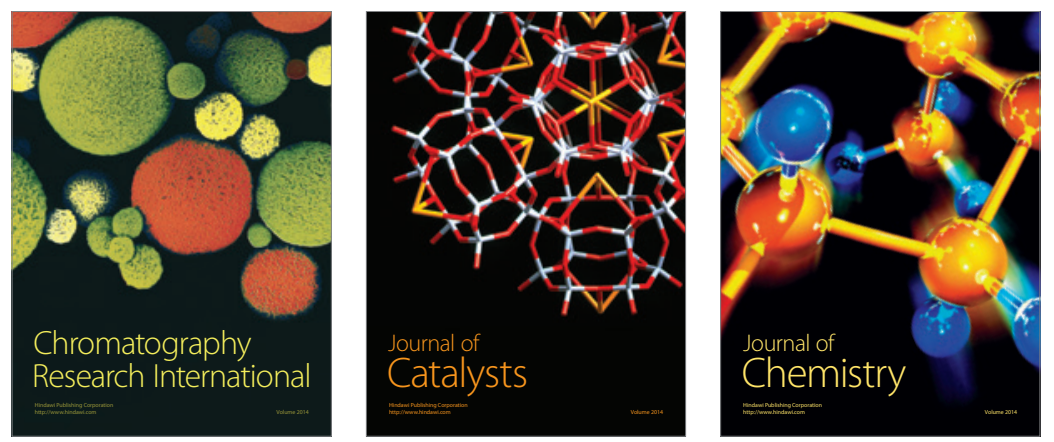
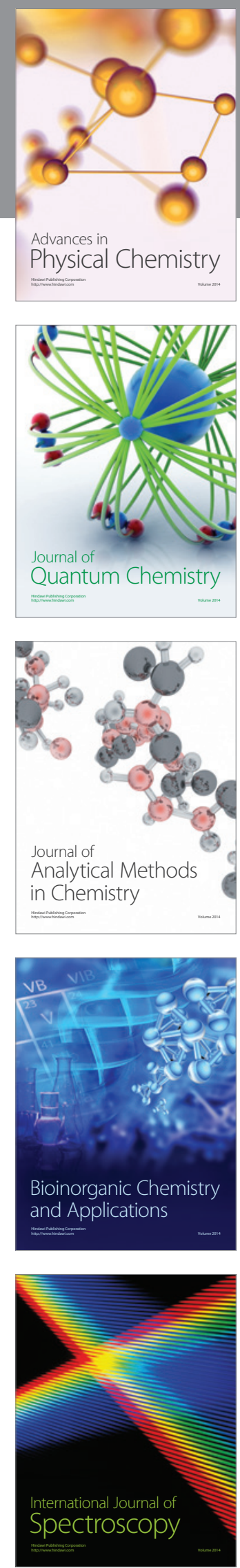